\title{
Ist die Verpflichtung zur Ausstrahlung von Wahlwerbung ein notwendiges Übel oder ist Wahlwerbung verzichtbar? - Eine rechtliche und politikwissenschaftliche Würdigung
}

\author{
Markus Vette
}

In dem vorliegenden Artikel soll zur Frage der Ausstrahlung von Wahlwerbesendungen durch die politischen Parteien im öffentlich-rechtlichen Rundfunk Stellung genommen werden. Dazu werden rechtliche und politikwissenschaftliche Argumente eingefuihrt, um die Frage zu klären, ob eine Verpflichtung zur Ausstrahlung von Wahlwerbesendungen im jeweiligen Landesmediengesetz relevant ist oder gegebenenfalls gestrichen werden kann.

\section{Ausgangsposition}

Die Wahlwerbung war und ist ein umstrittenes Thema in der öffentlichen Debatte der Bundesrepublik. Mehrfach war das Bundesverfassungsgericht mit Entscheidungen zur Wahlwerbung befaßt, so in der Entscheidung zur Wahlwerbung der Gewerkschaft im Betrieb ${ }^{1}$, zur Öffentlichkeitsarbeit der Bundesregierung im Jahre $1977^{2}$ und erneut $1983^{3}$, wo einem Staatsorgan die Wahlwerbung untersagt bzw. äußerste Zurückhaltung der Bundesregierung mit der Wahlbekanntmachung festgeschrieben wurde, und mit der Entscheidung zu Wahlwerbespots von $1978^{4}$, die in ihren Leitsätzen zur Argumentation herangezogen werden wird.

Nicht übersehen sollte man die Entscheidung zur Verteilung von Sendezeiten ${ }^{5}$ von 1983, wo im Vorfeld der Bundestagswahlen vom 6.3.1983 ein Wahlwerbespot der CSU im ZDF gesendet werden mußte.

\section{Rechtliche Würdigung}

Zunächst ist zu klären, ob den politischen Parteien ein subjektiv-öffentliches Recht zur Ausstrahlung von Wahlwerbung im öffentlich-rechtlichen Rundfunk zusteht. Das Recht politischer Parteien, Wahlwerbesendungen im öffentlich-rechtlichen Rundfunk auszustrahlen, wird maßgeblich durch den Artikel 5 des Grundgesetzes sowie den Artikel 21 Grundgesetz bestimmt. Artikel 5 regelt die Meinungsfreiheit, insbesondere die Programmfreiheit des Rundfunks. Artikel 21 GG charakterisiert die Stellung der Parteien im politischen System der Bundesrepublik:

In der Entscheidung BVerfGE 47, 198, $237^{6}$ wird bereits ausgesagt, daß es ein „unbeschränktes Zugangsrecht der politischen Parteien zu den Rundfunkanstalten“ nicht gibt und von Verfassungs wegen nicht geboten ist. In der juristischen Literatur wird dieser Auffassung kaum nennenswert widersprochen. ${ }^{7}$ Da es ein verfassungsunmittelbares subjektiv-öffentliches Recht auf die Ausstrahlung von Wahlwerbung im öffentlich-rechtlichen Rundfunk nicht gibt, bleibt es dem (einfachen) Gesetzgeber überlasen, den Parteien einen Anspruch auf Wahlwerbesendungen vor den Wahlen einzuräumen. In diesem Fasse muß der Gesetzgeber der Chancengleichheit ${ }^{8}$ Rechnung tragen und ermöglichen, daß alle Parteien diese Chance erhalten, wobei hinsichtlich der Dauer bzw. Anzahl der Wahlwerbespots durchaus einen Differenzierung vorgenommen werden kann. Dies ist die gegenwärtige Praxis.

Sollte der Gesetzgeber die in einem Landesgesetz bestehende Verpflichtung zur Ausstrahlung von Wahlwerbung streichen, könnten die Parteien grundsätzlich auf zwei Wegen dagegen vorgehen:

1. Ein Organstreitverfahren kann - da ein Bundesorgan ersichtlich nicht als Antragsgegner fungiert - allenfalls nach Maßgabe der Landesverfassung, d. h. vor dem betreffenden Landesverfassungsgericht, erfolgen. Dabei ist grundsätzlich zu beurteilen, ob es sich bei den betreffenden Grundrechten der Landesverfassung um wesensgleiche Bundesgrundrechte handelt.

2. Auf dem Wege einer Verfassungsbeschwerde kann eine Partei geltend machen, daß durch eine Verwaltungsmaßnahme (die Weigerung, die Wahlwerbung auszustrahlen) ihr Recht auf eine gleichberechtigte Benutzung einer Anstalt des öffentlichen Rechts verletzt worden sei. Auch diese Argumentation findet man in der oben genannten Entscheidung des Bundesverfassungsgerichtes. Für eine solche Vorgehensweise wären das Verfahren und die Fristen des Verfassungsgerichtsgesetzes zu beachten.

Ausgangspunkt der periodischen Diskussion um Wahlwerbesendungen ist immer wieder Kritik bzw. Empörung uiber mutmaßlich rechtswidrige Inhalte von solchen Sendungen: So wurde im Wahlkampf in Berlin 1995 bei einem Wahlwerbespot, der ausländische Mitbürger zeigte, die Musik „Spiel mir das Lied vom Tod“ unterlegt. ${ }^{9}$ Im Bundestagswahlkampf 1998 legte im Land Brandenburg die Christliche Liga ${ }^{10}$ Wahlwerbespots ${ }^{11}$ mit Texten vor, die deutliche Kritik im Rundfunkrat des ORB erfuhren. Die Aufzählung dieser Beispiele wäre durchaus beliebig fortsetzbar. Zunächst wurde 1995 in den ORB-Organen dazu geäußert, daß man nicht vor 
Beginn der Tagesschau einen Wahlwerbespot mit der Aufforderung zur Gewalt und 5 Minuten später in der Tagesschau Berichte über Gewalt und Ausschreitungen sehen sollte. - Kann ein Wahlwerbeverbot also die Lösung sein?

Wie ist die Verantwortung zu Wahlwerbespots z. Z. geregelt?

Gegenwärtig ist der Intendant mit der Aufgabe betraut, die Ausstrahlung von Wahlwerbung dann abzulehnen, wenn deren Inhalt offenkundig und schwerwiegend gegen die Gesetze verstößt. Diese Aufgabe soll jedoch nach Maßgabe der o. g. Entscheidung des Bundesverfassungsgerichtes ,in großzuigiger Handhabung“12 realisiert werden. Der Verstoß gegen Strafgesetze muß „evident"13 sein. - Tatsächlich führt eine Weigerung des Intendanten, einen Spot auszustrahlen, in der Regel zu einem Rechtsvorgang und zur Anordnung, die Aussendung des betreffenden Spots zu realisieren. ${ }^{14}$ Gerade durch diese öffentliche rechtliche Auseinandersetzung wird die Aufmerksamkeit auf den betreffenden Wahlwerbespot fokussiert, die Dilemmasituation beim Entscheidungsträger ist $\mathrm{m}$. E. offensichtlich.

Sollte die Verpflichtung zur Ausstrahlung der Spots aus dem Landesmediengesetz gestrichen werden, wäre $z$. B. die ausdrückliche Aufnahme eines Verbots von Wahlwerbesendungen ins Gesetz denkbar. Praktisch würde dies jedoch eine Beschränkung der Programmfreiheit des Rundfunks gemäß Artikel 5 des Grundgesetzes bedeuten. Man muißte der Argumentation begegnen, daß - nach der Meinung anderer - erwünschte und notwendige Beiträge zur allgemeinen Meinungsbildung untersagt wären.

Eine Verlagerung der Entscheidung uiber die Ausstrahlung von Wahlwerbesendungen in die Kompetenz des - die Interessen der Allgemeinheit vertretenden - Rundfunkrates läßt eine Gesetzgebung, die im Sinne einer „Gewaltenteilung“ eine Intendantenverfassung des öffentlich-rechtlichen Rundfunks kennt, wiederum nicht zu. Der Intendant bleibt verantwortlich. Da der Rundfunkrat ,über alle Fragen von grundsätzlicher Bedeutung" entscheidet und beschließt, dürfte seine Entscheidung auch hier - bei deren offensichtlich grundsätzlichem Charakter - dem konkreten Handeln und der Entscheidung des Intendanten zugrunde zu legen sein. (Die Vorstellung, daß der Rundfunkrat also von Fall zu Fall iiber die einzelnen Wahlwerbespots entscheidet, widerspricht den so geregelten Zuständigkeiten der Organe des öffentlich-rechtlichen Rundfunks.)

\section{Medienwissenschaftliche Gesichtspunkte}

Es gibt keine abschließenden Argumente aus der Medienwirkungsforschung dafuir, daß auf eine Aussendung von Wahlwerbespots verzichtet werden sollte: Untersuchungen über die Erzeugung der Aufmerksamkeit beim Bürger ergaben, daß große Plakate eher die Aufmerksamkeit auf bevorstehende Wahlen wecken können, als dies mit Wahlwerbespots der Fall ist. Ein jüngstes Beispiel fuir die Wirkung von Plakatierungen lieferte die Landtagswahl von Sachsen-Anhalt von 1998, die der rechtsgerichteten DVU mit intensivem Plakateinsatz einen Stimmenanteil von $13 \%$ brachte.

Da Wahlwerbespots im Fernsehen eher zufällig gesehen werden, erhöhen diese mehr die Bekanntheit der kleinen Parteien als die der großen Parteien. Der Wegfall der Wahlwerbespots würde damit den kleineren Parteien auch mehr schaden als den großen und bekannten Parteien. ${ }^{15}$

Gelegentlich findet man den Hinweis auf die angebliche wahlentscheidende Wirkung von Mediendarstellungen, speziell des Fernsehens. Gern wird dabei auf die Präsidentschaftswahl 1960 in den USA zwischen Kennedy und Nixon verwiesen, wo letzterer knapp unterlegen gewesen sein soll, zumal man danach das Umfrageergebnis erzielte, daß Nixon in der direkten Fernsehdebatte weniger Sympathien erhielt, da er wohl „schlecht rasiert“ war. - Angesichts des äußerst knappen Wahlergebnisses dieser Präsidentschaftswahl kann jedoch m. E. jedes denkbar andere Argument aus einer solchen Umfrage ebenso als "das wahlentscheidende“ Argument herangezogen werden. Es erscheint insgesamt fraglich, dem Fernsehen eine wahlentscheidende Wirkungsmächtigkeit zuzuordnen.

\section{Politikwissenschaftliche Würdigung}

Die Ausstrahlung von Wahlwerbespots durch die politischen Parteien ist nicht verfassungsunmittelbar, sondern durch das Landesmediengesetz geregelt. Sie ist und bleibt wesentlicher Bestandteil in der Phase des Wahlkampfes, um Aufmerksamkeit für die bevorstehende Wahl zu erzielen. Häufig wird die Bundesrepublik als Parteienstaat bezeichnet. Diese Parteien kämpfen um die Mehrheit, insbesondere bei Wahlen. ${ }^{16}$ Die Teilnahme der Parteien an Wahlen als „Kernstïck ihrer Tätigkeit" - so das Bundesverfassungsgericht - steht jedoch nicht im Widerspruch zu den Regelungen des Artikels 21 GG und des Parteiengesetzes, die insgesamt eine „Mitwirkung an der politischen Willensbildung des Volkes" beinhalten: Diese Mitwirkung ist in der Regel nicht umsetzbar allein innerhalb von 8 Wochen Wahlkampf, sondern eher von 4 oder 5 Jahren politischer, vor allem parlamentarischer Arbeit. Erneut kann auf das o. g. Beispiel von Sachsen-Anhalt verwiesen werden, wo etwa ein Jahr nach der Landtagswahl von 1998 die DVU-Fraktion zu zerfallen beginnt und nennenswerte parlamentarische Aktivitäten dieser Fraktion nicht zu verzeichnen sind ...

Eine Argumentation, daß die Aussendung von Wahlwerbespots das Kernstuick der Tätigkeit von politischen Parteien betrifft und deshalb zum Tabu erklärt wird, vermag somit nicht zu überzeugen.

Die Weigerung, Wahlwerbespots auszusenden, würde damit den Verzicht auf authentische Selbstdarstellung der politischen Parteien im Wettbewerb bedeuten. Eine Verlagerung der Entscheidungen über die tatsächliche Ausstrahlung vom Intendanten in den Rundfunkrat der öffentlich-rechtlichen Rundfunkanstalt ist durch die 
Zuständigkeiten der Organe der Anstalt nicht gedeckt, sie kann kaum im umfassenden Eigeninteresse der Parteien liegen:

Strittige Wahlwerbespots erfordern gerade die Auseinandersetzung und betreffen damit die originäre Aufgabe der politischen Parteien, an eben dieser politischen Willensbildung des Volkes mitzuwirken.

\section{Fazit}

So verlockend die Aussicht auf ein Verbot von Wahlwerbesendungen durch die politischen Parteien erscheint, so wenig vermag eine solche Verbotslösung der differenzierten Problemlage zu entsprechen, denn rechtlich wäre die Meinungsfreiheit durch - selbst umstrittene - verbotene, aber authentische Beiträge von Parteien beschränkt, von der Medienwirkungsforschung her stünden kleine Parteien hinsichtlich ihrer Chancengleichheit im Nachteil und politisch würde den Parteien eine wichtige Aufgabe, die Auseinandersetzung um die politische Willensbildung des Volkes gerade in Wahlkampfzeiten, „abgenommen werden“. Warum eigentlich?

Ideal wäre es sicher, wenn es weder vor Beginn der Tagesschau umstrittene Wahlwerbespots noch zu Beginn der Tagesschau Meldungen über etwaige Ausschreitungen gäbe. Politisch real ist, im Zweifel das kleinere Übel zu wählen. Dies ist dann sicher der Wahlwerbespot vor 20 Uhr, verbunden mit der erforderlichen gesellschaftspolitischen Debatte auch um die Inhalte dieser Spots, die von den Parteien gefuihrt werden muß.

\section{Anmerkungen}

[1] BVerfGE 42, 133-143 vom 28.04.1976

[2] BVerfGE 44, 125-197 vom 02.03.1977

[3] BVerfGE 63, 230-250 vom 23.02.1983

[4] BVerfGE 47, 198-239 vom 14.02.1978

[5] BVerfGE 63, 254-255 vom 28.02.1983

[6] BVerfGE 47, 198, 237 vom 14.02.1978

[7] Benda, 21.01.1999, Hauptausschuß des Landtags Brandenburg

[8] Dazu § 5 Parteiengesetz, veranlaßt durch Art. 3,1 und 3 GG sowie Art 21, 1, 1 GG

[9] SFB-Dokumentation

[10] ORB, Rundfunkrat

[11] Derselbe Vorgang fand im Kontext der Landtagswahlen 1999 in Brandenburg statt.

[12] BVerfGE 47, $198 \mathrm{ff}$, insbesondere Leitsatz 3

[13] BVerfGE 47, 198, 233

[14] Vgl. Entscheidung zum CSU-Spot vom 28.02.1983

[15] Wiedemann, 21.1.1999, Hauptausschuß des Landtags Brandenburg

[16] GG, Art. 28

\section{Literatur}

- BVerfGEntscheidungen

- GG

- Parteiengesetz

- Anhörung im Hauptausschuß des Landtages vom 21.01.1999

\section{Autor}

Der Autor ist Dozent für Staats- und Verfassungsrecht, Politikwissenschaft, Verwaltungslehre an der Technischen Fachhochschule Wildau. Er ist Verwaltungsratsvorsitzender des Ostdeutschen Rundfunks Brandenburg in Potsdam-Babelsberg.

\section{Dr. Dr. Markus Vette}

Dorfstraße 42 c

14476 Töplitz

Tel. (03 32 02) 60016

E-Mail:markus@Vette-net.de 